\title{
Systematische Bewertung von Auswirkungsanalysen des Engineering Change Managements
}

\author{
Iris Gräßler ${ }^{1 *}$, Dominik Wiechel*1 \\ ${ }^{1}$ Paderborn University, Heinz Nixdorf Institute, Chair for Product Creation \\ * Korrespondierender Autor: \\ Dominik Wiechel \\ Universität Paderborn \\ Heinz Nixdorf Institut \\ Lehrstuhl für Produktentstehung \\ Warburger Str. 100 \\ 33098 Paderborn \\ Telefon: 05251/606264 \\ Mail:dominik.wiechel@hni.upb.de
}

\begin{abstract}
Challenges in decisions on technical changes are the lack of knowledge about the expected impact and change propagation. Currently, no literature study contains a systematic differentiation and evaluation of existing approaches, which is a prerequisite for practitioners to select a suitable approach.

This research aims at defining differentiation criteria as well as generally applicable requirements for evaluation. A four-step approach is used: systematic literature review on approaches for impact analysis of engineering changes (1), categorization and prioritization of approaches based on reoccuring elements (2), derivation of context specific requirements for evaluation (3), and evaluation of approaches (4). The result indicates existing potential of object-oriented modeling approaches.
\end{abstract}

\section{Keywords}

Engineering Change Management, Impact Analysis, Engineering Changes, Model-based Systems Engineering, Product Development 


\section{Motivation}

Während der Entwicklung interdisziplinärer komplexer Gesamtsysteme sind technische Änderungen (Engineering Changes) unvermeidbar [1]. Die Auswirkungen der Änderungen können sich innerhalb des Systems und über die Systemgrenzen hinaus ausbreiten und zu ansteigenden Kosten oder Projektfehlschlägen führen [2]. Je komplexer ein System ist, desto mehr Abhängigkeiten bestehen zwischen den Sub-Systemen und Systemelementen, und desto schwieriger ist es, die Auswirkungen nachzuvollziehen und zu bewerten [3]. Der Management-Ansatz des Engineering Change Managements (ECM) umfasst unterstützende Methoden zur Organisation, Implementierung und Kontrolle von Änderungsbedarfen. Eine Herausforderung ist der unsystematische Umgang mit Änderungsbedarfen in der industriellen Praxis, obwohl Methoden und Hilfsmittel des ECM in der Literatur beschrieben werden [4]. Sofern ECM-Prozesse Anwendung finden, wird das Anwendungspotenzial nicht vollständig ausgeschöpft, da die unternehmensspezifische Anpassung (Tailoring) an die vorherrschenden Rahmenbedingungen in der Regel vernachlässigt wird. Beispiele sind die fehlende Zuweisung von Rollen und Verantwortlichkeiten sowie die fehlende zeitliche Terminierungen der Prozessschritte [5]. Eine weitere Herausforderung im Umgang mit Änderungen ist fehlendes Wissen über die zu erwartenden Auswirkungen und die Änderungsfortpflanzung [6]. Daraus resultiert eine unvollständige Informationsgrundlage für die Änderungsentscheidung. Darüber hinaus ist die Bewertung der Änderungsauswirkungen meistens ungenau und abhängig von Expertenwissen, ohne objektive Bewertungskriterien zugrundezulegen [4]. Vor allem die Vorhersage von Änderungspropagation stellt eine große Herausforderung dar [7]. Ansätze zur Auswirkungsanalyse im Kontext des ECM bieten eine Unterstützung für die Vorhersage von Änderungspropagation, indem Änderungen und ihre Propagationspfade systematisch identifiziert, abgebildet und bewertet werden [8]. Ein systematischer Vergleich von Ansätzen unter Berücksichtigung von Anforderungen aus der industriellen Praxis unterstützt Entwickler:innen bei Auswahl und bedarfsgerechter Anpassung der Ansätze und hilft die Herausforderungen des Engineering Change Managements (Bild 1) zu beherrschen.

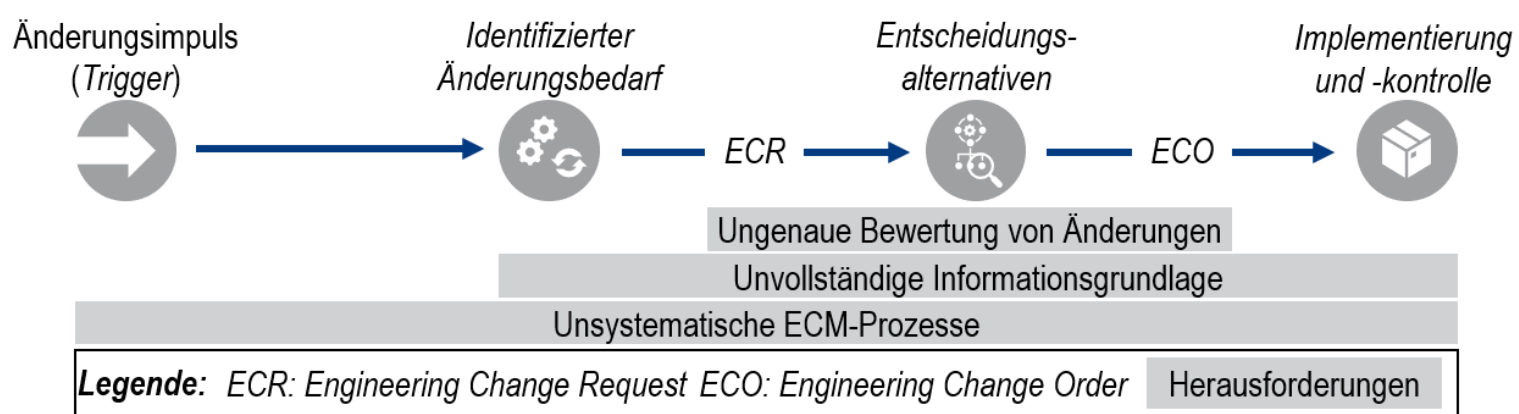

Bild 1: Herausforderungen des Engineering Change Managements

\section{Stand der Technik}

In einem komplexen technischen Gesamtsystem interagieren einzelne Subsysteme und Systemkomponenten miteinander, um das gewünschte Systemverhalten zu erzeugen und die Funktion des Systems zu ermöglichen [9]. Während der Entwicklung der Systeme entstehen Änderungsbedarfe. Engineering Changes (ECs) sind technische Änderungen und/oder Modifikationen der freigegebenen Struktur, des Verhaltens, der Funktionen oder der Beziehungen zwischen den Elementen eines technischen Artefakts [1]. Eine Literaturstudie [6] fokussiert sich auf ECs und ordnet die Begriffe in den Anwendungskontext ein: ECs werden durch Änderungsimpulse (Trigger) ausgelöst [6], anschließend identifiziert und durch den Änderungsantrag (Engineering Change Request) dokumentiert. Auf Basis unterschiedlicher Entscheidungsalternativen wird eine Entscheidung über die Ablehnung oder Genehmigung 
einer Änderung getroffen. Im Falle einer Genehmigung entsteht ein auszuführender Änderungsauftrag (Engineering Change Order) zur Umsetzung der Änderung, welche anschließend von Entwickler:innen implementiert und kontrolliert wird [10]. Unterschiedliche Ursachen können den Änderungsimplus auslösen, beispielsweise Flüchtigkeitsfehler, mangelnde Kommunikation, Änderungsausbreitung, Kosteneinsparungen sowie Vereinfachungen des Herstellungsprozesses oder Verbesserungen an dem zu entwickelnden System [11].

Das Änderungsmanagement (Engineering Change Management (ECM)) ist ein Management-Ansatz für die erfolgreiche Implementierung von Änderungen auf verschiedenen Systemebenen des zu entwickelnden Gesamtsystems sowie zur Bewertung der Auswirkungen von technischen Änderungen auf das Gesamtsystem [12]. Das Management umfasst Strategien und Maßnahmen zum Umgang mit technischen Änderungen und unterstützt die Verantwortlichen bei der Implementierung des ECs durch Prozessmodelle [13]. Ein Beispiel ist der generische Änderungsprozess nach JARRATT [14]. Im Engineering Change-Prozess müssen die entstehenden Kosten, der Nutzen sowie die Risiken einer möglichen Implementierung von Änderungen in Hinblick auf ihre Auswirkungen auf Zeitplan, Budget und Produktqualität abgewogen werden [15]. Unterschiedliche Literaturstudien adressieren das ECM: aufbauend auf initialen Studien zum ECM [16] definiert HAMRAZ ET AL. [1] ein Rahmenwerk, welches die Einordnung von Ansätzen aus dem Bereich ECM ermöglicht. Weitere Autoren vergleichen Ansätze des ECM anhand von definierten Anforderungen und entwickeln diese weiter [13, 17, 18].

Die Elemente sowie Abhängigkeiten zwischen Elementen können mithilfe einer ECAuswirkungsanalyse analysiert und bewertet werden. Eine Literaturstudie von JAYATILLEKE UND LAI [19] befasst sich mit existierenden Ansätzen im Bereich des Anforderungsmanagements. Nach BOHNER UND ARNOLD ist eine Auswirkungsanalyse die Aktivität zur Identifizierung dessen, was geändert werden muss, um eine Änderung zu erreichen, oder der Identifizierung der potenziellen Folgen einer Änderung [20]. Diese Definition aus dem Bereich Software-Entwicklung wird in diesem Beitrag für das ECM angepasst:

Die EC-Auswirkungsanalyse für das technische Änderungsmanagement ist die Aktivität der Identifikation potenzieller Effekte einer Änderung - einschließlich initialer und konsekutiver Effekte - und die Abschätzung der resultierenden (lokalen und kollektiven) Folgen, mit dem Ziel, eine effektive Entscheidung über Änderungsbedarfe zu unterstützen.

Der initiale Änderungsbedarf führt zur Änderungsauswirkungen, welche durch Entwicklungsentscheidungen über den Umfang und die Art der Änderung des betroffenen Elements determiniert werden. Die Auswirkungen werden analysiert, indem entstehende Effekte einer Änderung auf Elemente identifiziert und die daraus resultierenden Folgen abgeschätzt werden. Effekte beschreiben potenzielle Wirkungen der technischen Änderung auf die betroffenen Elemente (z. B. Strukturelemente). Die Effekte werden in zwei Arten unterteilt: Lokale Effekte beschreiben die Wirkung auf direkt betroffene Elemente. Konsekutive Effekte beschreiben die indirekten Wirkungen auf davon abhängige Elemente entlang von Propagationspfaden. Die anschließende Abschätzung der lokalen und kollektiven Folgen umfasst die Bewertung der erwarteten Auswirkungen. Lokale Folgen implizieren die Abschätzung der Auswirkungen auf direkt betroffene Systemelemente. Kollektive Folgen ergeben sich durch Aggregation der lokalen Folgen und aller indirekten nachfolgenden Änderungen entlang der Propagationspfade. Anhand der kollektiven Folgen können Entscheidungsalternativen verglichen werden (z. B. Ablehnung des Änderungsantrags oder Grad der Implementierung), wodurch effektive Entscheidungen getroffen werden können. Auswirkungen von Änderungsbedarfen können sowohl zu Einsparungen und Verbesserungen als auch zu Kostensteigerungen, Terminverzögerungen [12] und zu einer Reduzierung der Produktqualität [17] führen. 


\section{Forschungsproblem und Forschungsziele}

In der Literatur existieren unterschiedliche Ansätze, welche die Analyse von Auswirkungen technischer Änderungen ermöglichen. Bisherige Literaturstudien befassen sich jedoch entweder mit einem Überblick bestehender Lösungsansätze des übergeordneten Engineering Change Managements [1, 6, 16] oder fokussieren sich auf spezifische Aspekte, wie die Informationsmodelle [21] oder die unterstützenden Methoden [22]. Eine explizite Literaturstudie zur EC-Auswirkungsanalysen lässt sich durch die Fokussierung auf das Anforderungsmanagement nicht übertragen [19]. Keine Literaturstudie beinhaltet eine systematische kontextspezifische Differenzierung bestehender Lösungsansätze für ECAuswirkungsanalysen. Zusätzlich existiert keine vergleichbare Bewertung der Praxistauglichkeit von Lösungsansätzen, wodurch die Auswahl von Ansätzen sowie die zukünftige Optimierung der Ansätze erschwert wird. Forschungsbedarf besteht hier in der systematischen Herleitung allgemeingültiger, differenzierender Anforderungen an ECAuswirkungsanalysen. Daraus ergeben sich zwei Forschungsfragen, die in diesem Beitrag beantwortet werden:

Forschungsfrage 1 (RQ1): Welche Unterscheidungsmerkmale ermöglichen einen systematischen Vergleich von Ansätzen zur EC-Auswirkungsanalyse?

Forschungsfrage 2 (RQ2): Welche Anforderungen müssen zur vergleichenden Bewertung der Praxistauglichkeit von Lösungsansätzen zur EC-Auswirkungsanalysen abgeleitet werden?

\section{Verwendete Methoden bzw. Vorgehensweisen}

Die Forschungsfragen werden mithilfe eines vierschrittigen Vorgehens beantwortet, welches in Bild 2 dargestellt wird: Beginnend mit einer systematischen Literaturrecherche nach MACHI UND MCEVOY [23] werden Ansätze der EC-Auswirkungsanalyse in Google Scholar, Library der Design Society und Science Direct identifiziert (Schritt 1). Aus 1.101 vorselektierten wissenschaftlichen Dokumenten wurden 124 Dokumente mithilfe von KO-Kriterien (z.B. fehlender Bezug zu technischen Systemen) ausgewählt und schlussendlich 48 Ansätze als relevant eingestuft. Die Analyse und Kategorisierung der Ansätze wird anhand von fünf wiederkehrenden Elementen einer EC-Auswirkungsanalyse durchgeführt (Schritt 2). Je Kategorie werden Unterscheidungsmerkmale definiert, welche verschiedene Ausprägungen annehmen können. Mittels Zitationsanzahl werden je Kategorie drei repräsentative Ansätze ausgewählt. Parallel werden anhand aller 48 relevanten Ansätze explizit benannte und implizit berücksichtigte Anforderungen an EC-Auswirkungsanalysen abgeleitet sowie in einer Gesamtübersicht konsolidiert und kategorisiert (Schritt 3). Diese dienen als Bewertungsgrundlage für die Gegenüberstellung der repräsentativen Ansätze. Es wurden 95 Anforderungen abgeleitet, geclustert und auf 16 allgemeingültige Anforderungen der Praxistauglichkeit zurückgeführt. Diese 16 Anforderungen sind inhaltlich je einer der vier Anforderungskategorien nach HAMRAZ [17] zugehörig. Im letzten Schritt wird eine Abgrenzungsmatrix erstellt (Schritt 4), in der die Ansätze je Kategorie (Schritt 2) den abgeleiteten Anforderungen an die Auswirkungsanalyse (Schritt 3) gegenübergestellt werden. Die Ansätze werden anhand eines dreistufigen Schemas („erfüllt“, „teilweise erfüllt", "nicht erfüllt") bewertet. Die Bewertung ermöglicht einerseits die kritische Diskussion in Bezug auf die Praxistauglichkeit der Ansätze. Andererseits werden Entwickler:innen bei der unternehmensspezifischen Auswahl und Anpassung eines Ansatzes unterstützt. 


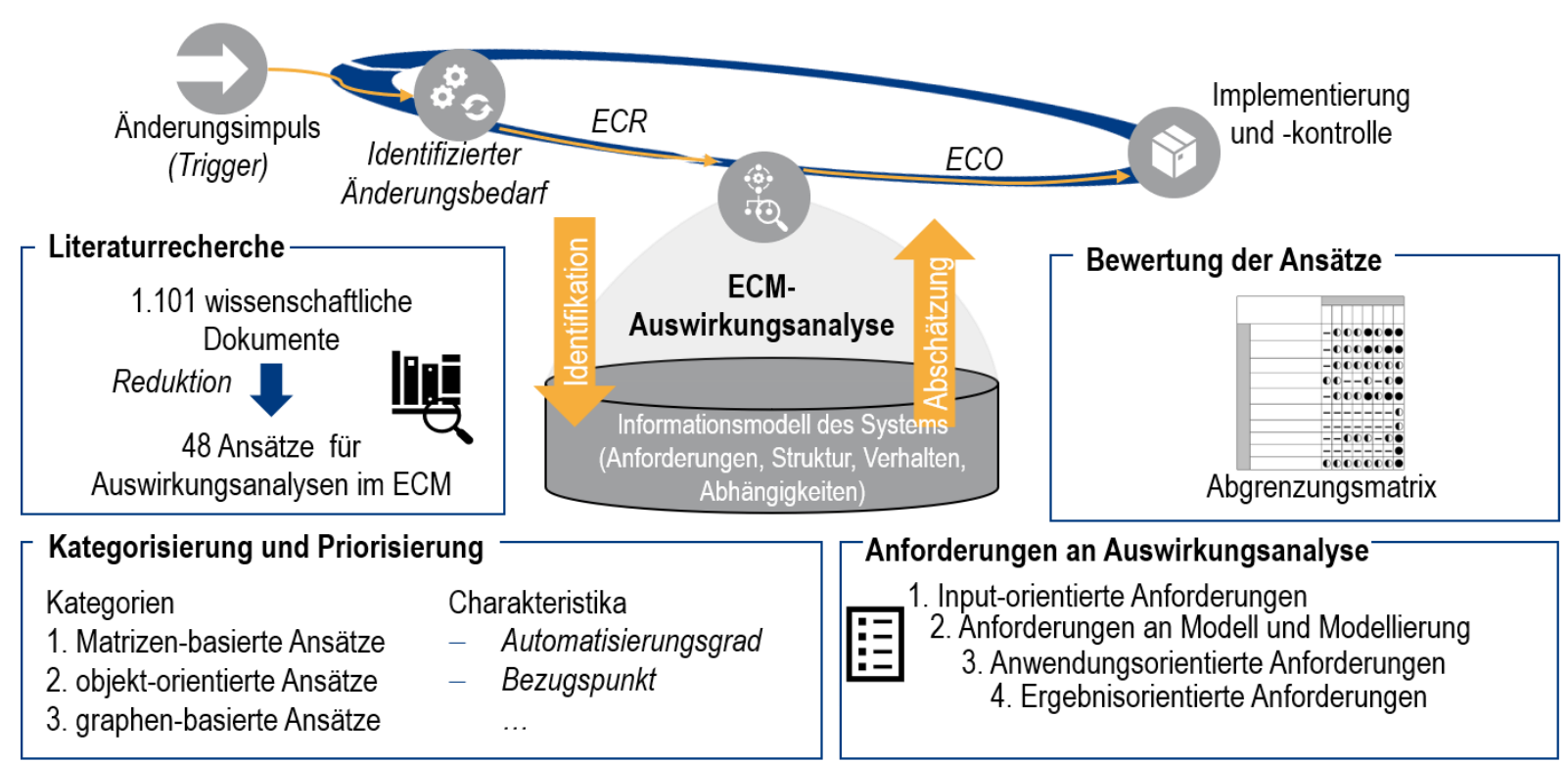

Bild 2: Systematische Bewertung von ECM-Auswirkungsanalysen

\section{Ergebnisse und Diskussion}

Die Analyse der 48 identifizierten Ansätze ermöglicht das Festlegen von fünf wiederkehrenden Elementen von EC-Auswirkungsanalysen. Zur weiteren Differenzierung werden die wiederkehrenden Elemente mit spezifischen Merkmalen konkretisiert, welche unterschiedliche Ausprägungen annehmen können. Die Merkmale sind durch die identifizierten Ansätze determiniert. Fünf wiederkehrende Elemente mit dazugehörigen Merkmalen und Ausprägungen wurden identifiziert:

1. Der Input wird charakterisiert durch die Art des ECs mit den Attributen Quelle, Richtung, Ebene, Formalität und Zeitpunkt [24].

2. Merkmale von Ansätzen zur Identifikation der Effekte eines ECs sind der Automatisierungsgrad des Ansatzes (nicht-automatisiert, teil-automatisiert, vollautomatisiert), die Tiefe der Analyse (initiale Effekte, konsekutive Effekte, kollektive Effekte) und der Umfang der Analyse (Art und Anzahl berücksichtigter Abhängigkeiten und Systemelemente z.B. Anforderungs-Element, Strukturelement, ...)

3. Das Informationsmodell wird durch die abgebildeten Modellelemente (Anzahl und Art), die Art des Informationsmodells (angelehnt an [13]: Matrix-basiert, Objektorientierte Modellierung, Graphen-basiert ) sowie die Abhängigkeitstypen (Richtung und Gewichtung) charakterisiert.

4. Die Abschätzung der Folgen wird durch die Merkmale des Automatisierungsgrads (nicht-automatisiert, teil-automatisiert, voll-automatisiert); der Nachvollziehbarkeit (nicht nachvollziehbar, teilweise nachvollziehbar, nachvollziehbar) und des Bezugspunkts der Abschätzung (Qualität, Kosten, Zeit) charakterisiert.

5. Der Output kann anhand des Merkmals der Ergebnisdimension (qualitatives Ergebnis, quantitatives Ergebnis) unterschieden werden.

Bild 3 verdeutlicht die Abhängigkeit der Identifikation der Effekte sowie der Abschätzung der Folgen von dem zugrundeliegenden Informationsmodell. Das Informationsmodell gilt aufgrund der Diversität von Abhängigkeiten zwischen unterschiedlichen Elementen der betrachteten technischen Systeme als bedeutender Faktor für aussagekräftige Auswirkungsanalysen [25]. 


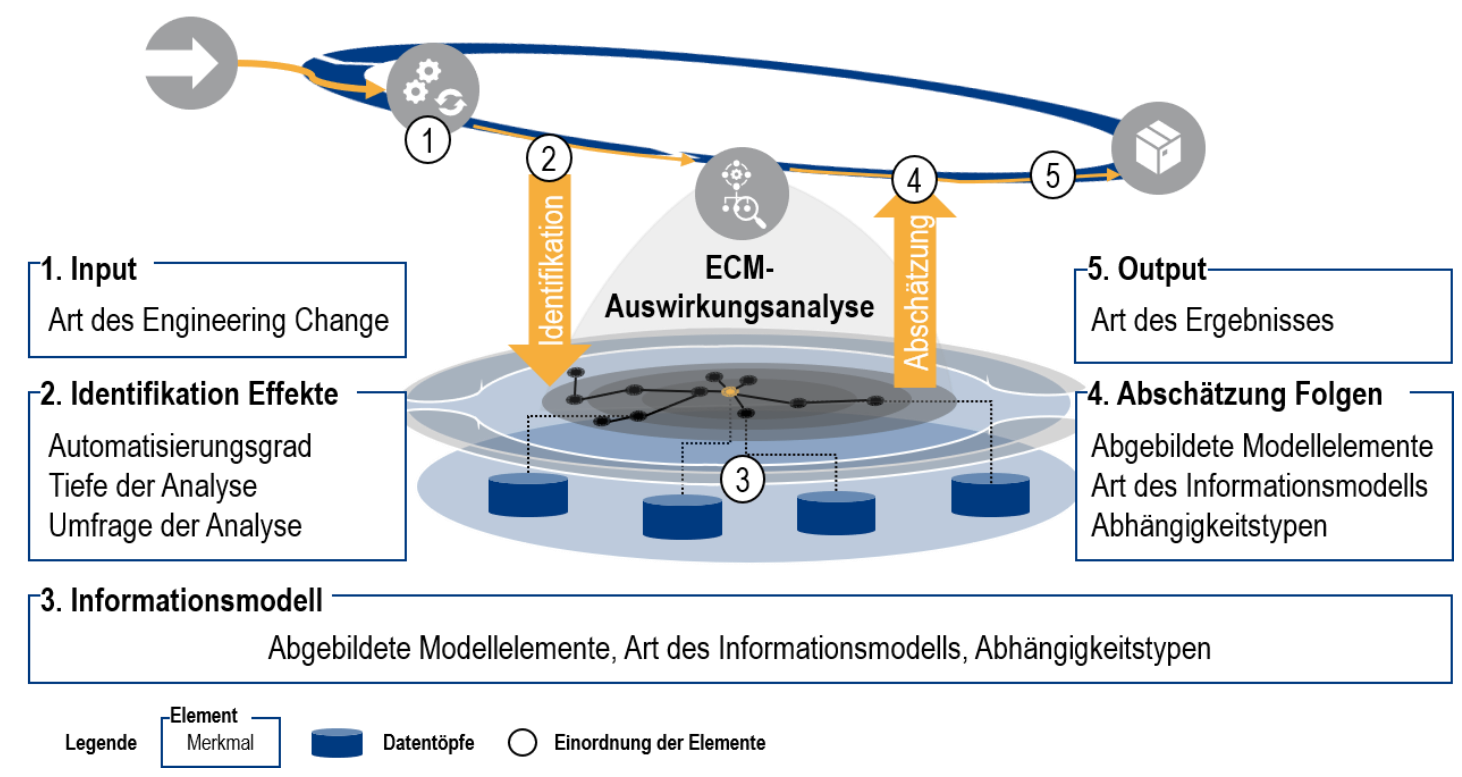

Bild 3: Wiederkehrende Elemente und Merkmale zur Differenzierzung von EC-Auswirkungsanalysen

Die 48 Ansätze werden daher nach den drei Kategorien des Merkmals Art des Informationsmodells aufgeteilt. Die Kategorien umfassen folgende Anzahl an Ansätzen: matrix-basierte Ansätze (17), objektorientierte Modellierungsansätze (8), graphen-basierte Ansätze (20). Wenn ein Ansatz auf mehr als ein Informationsmodell zugreift, wird das Informationsmodell zur Abschätzung der Folgen primär betrachtet, um eine eindeutige Zuordnung in die Kategorien sicherzustellen. Lediglich drei Ansätze können nicht in die Kategorien eingeordnet werden. Ein Beispiel ist der Ansatz CONRAD ET AL., in dem der Fokus auf eine tabellenförmige Fehlermöglichkeiten und Einflussanalyse (FMEA) gelegt wird [26]. Die abschließende Auwahl der drei repräsentativen Ansätze je Kategorie erfolgt anhand der durchschnittlichen Zitationszahl je Jahr, welche mithilfe des Einsehens der Zitationszahl auf der Literaturplattform Google Scholar errechnet wird. Die voneinander unabhängigen Ansätze werden in die Abgrenzungsmatrix übertragen (Schritt 4).

Im dritten Schritt werden allgemeingültige Anforderungen an die Praxistauglichkeit der EC-Auswirkungsanalyse definiert, welche ebenfalls in die Abgrenzungsmatrix (Schritt 4) übertragen werden. Die Anforderungen werden systematisch aus den 48 identifizierten Ansätzen hergeleitet. Neben explizit benannten Anforderungen an EC-Auswirkungsanalysen werden auch implizite Anforderungen zur Praxistauglichkeit erhoben, beispielsweise aus den Abschnitten Diskussion und Ausblick. Die 95 Anforderungen aus der Analyse der Ansätze wurden auf Basis der Erkenntnisse aus Schritt 2 geclustert, um allgemeingültige Anforderungen der Praxistauglichkeit abzuleiten. Nachfolgend werden die 16 Anforderungen der Praxistauglichkeit anhand der Anforderungskategorien nach Hamraz [17] eingeordnet:

- Input-orientierte Anforderungen: Die Auswirkungsanalyse muss so gestaltet sein, dass Sie mit vorhandenen Informationen und Daten durchgeführt werden kann [13]. Der Änderungsbedarf muss sich auf unterschiedliche Elemente beziehen können (Anforderungen, Strukturelemente, Verhaltenselemente) [17, 27].

- Anforderungen an Modell und Modellierung: Die Auswirkungsanalyse muss Ausbreitungspfade der Änderungspropagation darstellen [17, 18, 28]. Die Auswirkungsanalyse muss Propagationseffekte berücksichtigen [29, 30]. Teile des Informationsmodells müssen wiederverwendet werden können [4]. Die Auswirkungsanalyse muss Informationen aus disziplinspezifischen Partialmodellen integrieren [4, 13, 31-33]. Das Informationsmodell muss Informationen auf verschiedenen Granularitätsleveln (Tiefe) und aus verschiedenen Disziplinen (Breite) bereistellen [13, 32-34]. 
- Anwendungsorientierte Anforderungen: Der zeitliche Anwendungsaufwand der Auswirkungsanalyse muss in der industriellen Praxis akzeptabel sein [35]. Die Auswirkungsanalyse muss (teil-)automatisiert durchführbar sein [4, 36]. Die Software zur Durchführung der Auswirkungsanalyse muss frei oder kommerziell verfügbar sein [4, 17]. Die Ergebnisse der Auswirkungsanalye müssen an die unternehmensspezifischen Bedarfe angepasst werden können (Tailoring [37]). Die Datenauswertung und Bereitstellung muss akteurübergreifend (z.B. mit Zulieferern) möglich sein [4, 38, 39].

- Ergebnisorientierte Anforderungen: Die Ergebnisse der Auswirkungsanalyse müssen eine hinreichende Genauigkeit vorweisen und die qualitativen Ansprüche hinreichend erfüllen [13]. Die Ergebnisse der Auswirkungsanalyse sollen nachvollziehbar und transparent sein [40]. Die entstehenden Kosten einer potenziellen Änderung müssen anhand vorhandener Informationen bewertbar sein [2, 31, 41, 42]. Die Auswirkungsanalyse muss auf unterschiedliche Unternehmensgrößen skalierbar sein, um in der industriellen Praxis zum Einsatz zu kommen [18].

Tabelle 2: Abgrenzungsmatrix zum Vergleich repräsentativer Ansätze

\begin{tabular}{|c|c|c|c|c|c|c|c|c|c|}
\hline \multirow[b]{2}{*}{ Anforderung } & \multicolumn{3}{|c|}{$\begin{array}{l}\text { Matrix-basierte } \\
\text { Ansätze }\end{array}$} & \multicolumn{3}{|c|}{$\begin{array}{l}\text { Objektorientierte } \\
\text { Modellierungs- } \\
\text { ansätze }\end{array}$} & \multicolumn{3}{|c|}{$\begin{array}{c}\text { Graphen-basierte } \\
\text { Ansätze }\end{array}$} \\
\hline & $\Xi$ & $\begin{array}{l}\infty \\
m\end{array}$ & $\stackrel{\infty}{ \pm}$ & $\overline{\bar{m}}$ & $\underset{\Xi}{\Xi}$ & m & Ð্ণ & $\stackrel{n}{\square}$ & $\stackrel{\sqrt[5]{ \pm}}{ \pm}$ \\
\hline \multicolumn{10}{|c|}{ 1.1 Verfügbarkeit von Daten / Informationen } \\
\hline \multicolumn{10}{|c|}{ 1.2 Unterschiedliche Arten der Änderung } \\
\hline \multicolumn{10}{|c|}{ 2.1 Modellierung von Ausbreitungspfaden } \\
\hline \multicolumn{10}{|c|}{ 2.2 Propagationseffekte berücksichtigen } \\
\hline \multicolumn{10}{|l|}{ 2.3 Wiederverwendbarkeit } \\
\hline \multicolumn{10}{|l|}{$\begin{array}{l}2.4 \text { Integration vorhandener } \\
\text { Informationsmodelle }\end{array}$} \\
\hline \multicolumn{10}{|c|}{ 2.5 Tiefe und Breite des Informationsmodells } \\
\hline \multicolumn{10}{|c|}{ 3.1 Zeitlicher Anwendungsaufwand } \\
\hline \multicolumn{10}{|l|}{ 3.2 Automatisierbarkeit } \\
\hline \multicolumn{10}{|c|}{ 3.3 Verfügbarkeit von Software } \\
\hline \multicolumn{10}{|c|}{ 3.4 Tailoring / Anwenderindividuelle Analyse } \\
\hline \multicolumn{10}{|c|}{ 3.5 Akteurübergreifende Kollaboration } \\
\hline \multicolumn{10}{|l|}{ 4.1 Ergebnisqualität } \\
\hline \multicolumn{10}{|l|}{ 4.2 Nachvollziehbarkeit } \\
\hline \multicolumn{10}{|l|}{ 4.3 Bewertung der Kosten } \\
\hline \multicolumn{10}{|l|}{4.4 Industrielle Anwendbarkeit } \\
\hline Summe & 6 & 5,5 & 5,5 & 12 & 12 & 11 & 11 & 10,5 & 11,5 \\
\hline
\end{tabular}

Tabelle 2 illustriert die Abgrenzung und Bewertung der repräsentativen Ansätze. Die Bewertung der Ansätze basiert auf der Einschätzung der Autoren. Die anschließende Diskussion der Evaluationergebnisse wird nach den Arten des Informationsmodells aufgeteilt. 
Die matrix-basierten Ansätze sind nach der Anzahl der Zitationen weit verbreitet. In Matrizen können unterschiedliche Abhängigkeitstypen nachvollziehbar dargestellt werden. Die Ansätze unterliegen jedoch Limitationen, wodurch die Bewertung lediglich zwischen 5,5 und 6 liegt. Die Schritte der Vorgehensweise sind sequentiell vorgegeben und können nicht an die individuellen Interessen der Anwender:innen im Sinne des Tailorings angepasst werden. Die Ansätze werden nicht durch Software-Anwendungen unterstützt, wodurch ein erhöhter zeitlicher Aufwand für das manuelle Befüllen der Matrizen entsteht. Die Matrizen sind in sich geschlossene Modelle, die keine Integration anderer Partialmodelle ermöglichen.

Die Summe der erfüllten Anforderungen der objektorientierten Modellierungsansätze ist mit bis zu 12 Punkten am höchsten. Bei Ansätzen der objektorientierten Modellierung werden unterschiedliche Modellierungssprachen verwendet, um Informationsmodelle für die ECAuswirkungsanalyse zu modellieren. Beispiele sind UML [44], SysML [31] und EXPRESS [33]. Das Informationsmodell ist weder in der Tiefe noch in der Breite begrenzt. Die Ausbreitungspfade und Propagationseffekte werden durch die Modellelemente und Abhängigkeiten berücksichtigt. Hinzukommend ergeben sich Möglichkeiten der vollständigen Automatisierbarkeit, beispielsweise durch die Definition von Zusicherungen in der Object Constraint Language [44]. Weitere objektorientierte Modellierungs-Ansätze [25] unterstreichen die genannten Potenziale durch die Validierungsergebnisse. Optimierungspotenzial liegt in der Ergebnisqualität der Auswirkungsanalysen sowie im Anwendungsaufwand. Beides kann durch die Wiederverwendung von Modellen verbessert werden.

Die Graphenbasierten Ansätze erreichen ähnlich hohe Summenwerte $(11$ bis $11 ., 5)$ bei der Erfüllung der Anforderungen. Graphen-basierte Ansätze können ebenfalls von der Tiefe und Breite an die benötigten Informationsbedarfe angepasst werden. Die Informationsmodelle bieten die Möglichkeit, Auswirkungsanalysen automatisiert durchzuführen. Die akteurübergreifende Kollaboration ist limitiert. Die Integration vorhandener disziplinspezifischer Informationsmodelle wird nur in Einzelfällen ermöglicht.

Informationsmodell-übergreifende Defizite bestehen bei der Bewertung der Kosten sowie bei der Integration unterschiedlicher disziplinspezifischer Partialmodelle. Ein weiteres Defizit ist der zeitliche Anwendungsaufwand, welcher bei allen Ansätzen durch die Wiederverwendung von Informationsmodellen erhöht werden kann. Hinzukommend ist die Verfügbarkeit von Daten und Informationen beschränkt, da die benötigten Informationsmodelle einerseits zusätzlich erstellt werden müssen und andererseits auf subjektivem Expertenwissen beruhen. Das subjektive Expertenwissen führt zu einer reduzierten Ergebnisqualität Diese könnte durch die automatisierte Analyse objektiver Daten eines vollständigen Informationsmodells verbessert werden. Ein Beispiel sind algorithmenbasierte Auswirkungsanalysen, welche auf Parameter unterschiedlicher Partialmodelle zugreifen. Lediglich bei den Modellen der objektorientierten Modellierung ergeben sich Synergieeffekte, beispielsweise durch vorhandene Systemmodelle aus der modellbasierten Systementwicklung sowie durch die Möglichkeite der akteurübergreifenden Modellierung.

\section{Zusammenfassung und Ausblick}

In diesem Beitrag wird eine systematische Literaturanalyse durchgeführt, um Entscheidungsmerkmale zur Charakterisierung von EC-Auswirkungsanalysen zu definieren und allgemeingültige Anforderungen an die Praxistauglichkeit herzuleiten. Das Ergebnis der systematischen Bewertung ermöglicht zum einen die bedarfsgerechte Selektion existierender Lösungsansätze. Zum anderen werden Defizite und Handlungsbedarfe identifiziert, um zielgerichtete Forschung im Bereich ECM-Auswirkungsanalysen zu ermöglichen und eine Effizienzsteigerung in der Produktentwicklung komplexer Systeme zu erreichen. Die Forschungsfragen werden beantwortet, indem wiederkehrende Elemente und Merkmale von EC-Auswirkungsanalysen identifiziert werden, welche einen systematischen Vergleich der Ansätze ermöglichen (RQ1). Hinzukommend werden allgemeingültige praxisorientierte 
Anforderungen an EC-Auswirkungsanalysen abgeleitet (RQ2). Die abschließende praxisorientierte Bewertung und Gegenüberstellung in der Abgrenzungsmatrix anhand der einzelnen Tabelleneinträge belegt das Differenzierungspotenzial der abgeleiteten Anforderungen. Inhaltlich wird das Potenzial objekt-orientierter Modellierungssprachen für ECAuswirkungsanalysen deutlich. Im Vergleich zu matrix- und graphen-basierten Ansätzen ermöglichen es diese, mittels Erweiterung des Systemmodells Abhängigkeiten simultan zur Entwicklung des Systemmodells abzubilden. Ebenso ergeben sich Synergieeffekte durch die modellbasierte Systementwicklung, da die erfolgreiche Implementierung einer ECAuswirkungsanalyse ein effektives Engineering Change Management im Systemmodell ermöglicht [46]. Daher ergibt sich zusätzlicher Forschungsbedarf in der Verbesserung der Integration von Parametern sowie bei der Unterstützung der Durchführung von ECAuswirkungsanalysen z. B. durch unternehmensspezifische Rollenmodelle [47].

Durch die Differenzierung der repräsentativen Ansätze zur EC-Auswirkungsanalyse sowie deren anforderungsbasierte Evaluation wird die bedarfsgerechte Auswahl eines Lösungsansatzes in der Praxis unterstützt und Forschungsbedarfe aufgezeigt. So wird ein effizienterer Umgang mit technischen Änderungen in der Entwicklung komplexer Systeme ermöglicht.

\section{Literaturverzeichnis}

[1] Hamraz, Bahram; Caldwell, Nicholas H. M.; Clarkson, P. John: A Holistic Categorization Framework for Literature on Engineering Change Management. In: Systems Engineering 16 (2013), 4, S. 473-505.

[2] Eger, Tido; Eckert, Claudia M.; Clarkson, P. John: Engineering Change Analysis during Ongoing Product Development. In: DS 42: Proceedings of ICED (2007), S. 629-630.

[3] International Council on Systems Engineering (INCOSE): Systems Engineering Vision 2025. (2014).

[4] lakymenko et al.: Managing engineering changes in the engineer-to-order environment: challenges and research needs. In: IFAC-PapersOnLine 51 (2018), S. 144-151.

[5] Hölttä et al.: Lean information management model for engineering changes. In: World Academy of Science, Engineering and Technology 42 (2010), S. 1459-1466.

[6] Jarratt et al.: Engineering change: an overview and perspective on the literature. In: Research in Engineering Design 22 (2011), S. 103-124.

[7] Clarkson, P. John; Simons, Caroline; Eckert, Claudia M.: Predicting Change Propagation in Complex Design. In: Journal of Mechanical Design 126 (2004) 5, S. 788-797.

[8] Bohner, Shawn A.; Arnold, Robert S.: Software change impact analysis. Los Alamitos: Wiley-IEEE Computer Society Press, 1996.

[9] Eckert, Claudia M.; Zanker, Winfried; Clarkson, John P.: Aspects of a better understanding of changes. In: International Conference On Engineering Design ICED'01 (2001), S. 147-154.

[10] Monahan, R. Elaine: Engineering documentation control practices and procedures. Marcel Dekker Inc, 1995.

[11] Lee et al.: Capturing and reusing knowledge in engineering change management: A case of automobile development. In: Information Systems Frontiers 8 (2006) 5, S. 375-394.

[12] Fricke et al.: Coping with changes: Causes, findings, and strategies. In: Systems Engineering 3 (2000) 4, S. 169-179.

[13] Wickel, Martina Carolina: Änderungen besser managen - Eine datenbasierte Methodik zur Analyse technischer Änderungen, München: Technische Universität München, 2017.

[14] Jarratt, Timothy; Clarkson, P. John; Eckert, Claudia: Engineering change. In: Clarkson, John P.; Eckert, Claudia (Hrsg.) Design process Improvement., London, Springer, 2007, S. 262-285.

[15] Pasqual, Michael C.; de Weck, Olivier L.: Multiplayer Network Model for Analysis and Management of Change Propagation. In: International Conference on Engineering Design ICED11 (2011), S. 126-138.

[16] Wright, lan C.: A review of research into engineering change management: implications for product design. In: Design Studies 18 (1997) 1, S. 33-42.

[17] Hamraz, Bahram: Engineering Change Modelling Using a Function-Behaviour-Structure Scheme. Cambridge: LAP LAMBERT Acamdemic Publishing, 2013.

[18] Lemmens, Yves Claude Jean: Modelling and Analysis of Engineering Changes in Complex Systems. Cranfield: Cranfield University 2007.

[19] Jayatilleke, Shalinka; Lai, Richard: A systematic review of requirements change management. In: Information and Software Technology 93 (2018), S. 163-185. 
[20] Arnold, Robert S.; Bohner, Shawn A.: Impact analysis-Towards a framework for comparison. In: 1993 Conference on Software Maintenance (1993), S. 292-301.

[21] Ahmad, Naveed; Wynn, David C.; Clarkson, John P.: Information Models used to manage engineering change: A review of the Literature 2005-2010. In: International Conference on Engineering Design ICED11 (2011), S. 538-549.

[22] Ullah, Inayat; Tang, Dunbing; Yin, Leilei: Engineering Product and Process Design Changes: A Literature Overview. In: Procedia CIRP 56 (2016), S. 25-33.

[23] Machi, Lawrence. A.; McEvoy, Brenda T.: The literature review. Six steps to success. Thousand Oaks: Corwin Press, 2012.

[24] Kalpinen, Malia. S.; Eckert, Claudia; Clarkson, John P.: Assessing Impact Analysis practice to improve change management capability. In: Internation Conference of Engineering Design ICED'09 (2009).

[25] Wilms et al.: Using a cross domain product model to support engineering change management. In: Proceedings of the Design Society DESIGN Conference 1 (2020), S. 1165-1174.

[26] Conrad et al.: Change impact and risk analysis (CIRA)-combining the CPM/PDD theory and FMEAmethodology for an improved engineering change management. In: 16th International Conference on Engineering Design ICED 07 (2007).

[27] Ma, Yongsheng; Chen, Gang; Thimm, Georg: Change propagation algorithm in a unified feature modeling scheme. In: Computers in Industry 59 (2008), S. 110-118.

[28] Ahmad, Naveed; Wynn, David C.; Clarkson, John P.: Development and evaluation of a tool to estimate the impact of design change. In: Proceedings of DESIGN 2010 (2010), S. 105-116.

[29] Morkos, Beshoy; Shankar, Prabhu; Summers, Joshua David: Predicting requirement change propagation, using higher order design structure matrices: an industry case study. In: Journal of Engineering Design 23 (2012).

[30] Cohen, Tal; Fulton, Robert E.: A Data Approach to Tracking and Evaluating Engineering Changes. In: 12th Engineering Information Management Symposium 7 (1998).

[31] Fei et al:: A method for engineering design change analysis using system modelling and knowledge management techniques. In: International Journal of Computer Integrated Manufacturing 24 (2011) 6, S. 535-551.

[32] Hamraz et al.: Requirements-based development of an improved engineering change management method. In: Journal of Engineering Design 24 (2013) 11, S. 765-793.

[33] Cohen, Tal.; Navathe, S. B.; Fulton, Robert E.: C-FAR, change favorable representation. In: Computer-Aided Design 32 (2000) 5-6, S. 321-338.

[34] Cheng, Hui; Chu, Xuening: A network-based assessment approach for change impacts on complex product. In: Journal of Intelligent Manufacturing 23 (2012) 4, S. 1419-1431.

[35] Kattner et al.: A method for the expert-based identification of engineering change propagation. In: Proceedings of the 21st International Conference on Engineering Design 4 (2017), S. 633-642.

[36] Ouertani, Mohamed. Z.; Gzara-Yesilbas, Lilia; Lossent, Luc: Engineering Change Process: State of the Art; a case study and proposition of a impact analysis method. In: IDMME. (2004).

[37] Walden et al.: Systems engineering handbook. A guide for system life cycle processes and activities. New Jersey: Wiley \& Sons Inc., 2015.

[38] Köhler et al.: A matrix representation of the CPM/PDD approach as a means for change impact analysis. Om: Proceedings DESIGN (2008), S. 167-174.

[39] Reddi, Krishna R.; Moon, Young B.: System dynamics modeling of engineering change management in a collaborative environment. In: The International Journal of Advanced Manufacturing Technology 55 (2011) S. 1225-1239.

[40] Graessler, Iris; Oleff, Christian; Scholle, Philipp: Method for Systematic Assessment of Requirement Change Risk in Industrial Practice. In: Applied Sciences 10 (2020) 23, S. 86-97.

[41] Oduguwa, Patrick A.: Cost impact analysis for requirements management, Cranfield: Cranfield University, 2007.

[42] Rebentisch et al.: Assessment of changes in engineering design using change propagation cost analysis. In: DS 87-4 Proceedings of the 21st International Conference on Engineering Design (2017), S. 69-78.

[43] Flanagan et al.: Functional analysis of change propagation. In: Proceedings of the 14th International Conference on Engineering Design (2003).

[44] Briand et al.: Automated impact analysis of UML models. In: Journal of Systems and Software 79 (2006) 3, S. 339-352.

[45] Yang, Fan; Duan, Gui-jiang: Developing a parameter linkage-based method for searching change propagation paths. In: Research in Engineering Design 23 (2012), S. 353-372.

[46] Wang, Haoqi; Thomson, Vincent J.; Tang, Chengtong: Change propagation analysis for system modeling using Semantic Web technology. In: Advanced Engineering Informatics 35 (2018), S. 17-29.

[47] Gräßler, Iris; Wiechel, Dominik; Pottebaum, Jens: Role model of model-based systems engineering application. In: IOP Conference Series: Materials Science and Engineering (2021). 